\title{
Répartition des valeurs d'une classe de fonctions multiplicatives
}

par

A. Smati (Limoges)

1. Introduction. Soit $G$ la classe des fonctions multiplicatives $g$ ayant les propriétés suivantes :

(1) $g(n)>0$ pour tout entier $n \geq 1$.

(2) Il existe un nombre réel $\delta>0$ tel que pour tout nombre premier $p$

$$
g(p)=1+O\left(p^{-\delta}\right) .
$$

(3) Il existe un nombre réel $0<b<1 / 2$ tel que pour tout entier $n \geq 1$

$$
g(n) \gg n^{-b} .
$$

E. J. Scourfield [5] a montré, entre autres choses, le théorème suivant :

ThÉORÈme 1. Soit $g \in G$. Il existe une constante

$$
A(g)=\prod_{p}\left(1+\sum_{\alpha=1}^{\infty} p^{-\alpha}\left(\frac{1}{g\left(p^{\alpha}\right)}-\frac{1}{g\left(p^{\alpha-1}\right)}\right)\right),
$$

telle que, pour $x \rightarrow+\infty$, on ait

$$
\sum_{n g(n) \leq x} 1=A(g) x+O_{\varepsilon}(x \exp (-(1-\varepsilon) \sqrt{(\delta / 2) \log x \log \log x}))
$$

pour tout nombre réel $\varepsilon>0$.

Ensuite, elle a décrit une procédure permettant d'obtenir les résultats suivants comme conséquence du théorème $1, \varphi(n)$ désignant la fonction d'Euler et $\sigma_{\nu}$ la fonction

$$
\sigma_{\nu}(n)=\sum_{d \mid n} d^{\nu}, \quad \nu>0 .
$$

Corollaire 1. On a, pour tout $\varepsilon>0$,

(a) $\sum_{n(\varphi(n))^{\gamma} \leq x} 1=A_{1} x^{1 /(1+\gamma)}$ 


$$
+O_{\varepsilon}\left(x^{1 /(1+\gamma)} \exp \left(-(1-\varepsilon) \sqrt{\frac{1}{2(1+\gamma)} \log x \log \log x}\right)\right)
$$

où $\gamma>-1$ et $\gamma \neq 0$,

(b)

$$
\begin{array}{rl}
\sum_{n\left(\sigma_{\nu}(n)\right)^{\gamma} \leq x} & 1=A_{2} x^{1 /(1+\nu \gamma)} \\
+ & O_{\varepsilon}\left(x^{1 /(1+\nu \gamma)} \exp \left(-(1-\varepsilon) \sqrt{\frac{\nu}{2(1+\nu \gamma)} \log x \log \log x}\right)\right)
\end{array}
$$

où $\nu>0, \gamma>-1 / \nu, \gamma \neq 0$.

La méthode utilisée pour la démonstration du théorème 1 est analytique. En fait, c'est exactement la méthode C qu'utilisa P. T. Bateman [2] pour obtenir le cas particulier suivant de ce théorème 1 :

$$
\sum_{\varphi(n) \leq x} 1=\frac{\zeta(2) \zeta(3)}{\zeta(6)} x+O_{\varepsilon}(x \exp (-(1-\varepsilon) \sqrt{(1 / 2) \log x \log \log x}))
$$

pour tout $\varepsilon>0, \zeta$ étant la fonction de Riemann.

M. Balazard et l'auteur [1] ont retrouvé (1) par des méthodes élémentaires, c'est-à-dire, fondées sur des arguments n'utilisant pas l'intégration dans le plan complexe. L'auteur [6], [7] a également généralisé (1) à une classe de fonctions multiplicatives définies par A. Ivić [4].

On se propose ici de donner une démonstration d'une version légèrement plus faible du théorème 1 où dans le terme d'erreur $\delta$ est remplacé par $\beta=\min \{1, \delta\}$. Mais notre démonstration est, en revanche, élémentaire. Elle repose sur le théorème suivant qui possède un intérêt intrinsèque.

ThÉORÈme 2. On a, uniformément pour q tel que $0 \leq \log q \leq\left(\frac{1}{4} \log x\right)^{2}$,

$$
\begin{aligned}
M_{q}(x) & =: \sum_{\substack{n g(n) \leq x \\
(n, q)=1}} \mu^{2}(n) \\
& =B(q, g) x+O_{\varepsilon}(x \exp (-(1-\varepsilon) \sqrt{(\beta / 2) \log x \log \log x}))
\end{aligned}
$$

où $\mu$ désigne la fonction de Möbius, $\beta=\min \{1, \delta\}$, $\varepsilon$ réel positif arbitraire et

$$
B(q, g)=\prod_{p \mid q}\left(1+\frac{1}{p g(p)}\right)^{-1} \prod_{p}\left(1-\frac{1}{p}\right)\left(1+\frac{1}{p g(p)}\right),
$$

p étant un nombre premier générique.

On a, par exemple, le corollaire suivant du théorème 2 :

Corollaire 2. On a, pour tout $\varepsilon>0$,

(a)

$$
\sum_{\varphi(n) \leq x} \mu^{2}(n)=x+O_{\varepsilon}(x \exp (-(1-\varepsilon) \sqrt{(1 / 2) \log x \log \log x})),
$$


(b) $\quad \sum_{\sigma(n) \leq x} \mu^{2}(n)=\prod_{p}\left(1-\frac{2}{p(p+1)}\right) x$

$$
+O_{\varepsilon}(x \exp (-(1-\varepsilon) \sqrt{(1 / 2) \log x \log \log x})) .
$$

Le reste de l'article est organisé comme suit : le paragraphe 2 est consacré aux notations, le 3 à la démonstration de notre version du théorème 1 comme conséquence du théorème 2 et enfin les paragraphes 4 et 5 à la démonstration du théorème 2 .

Je remercie vivement Aleksandar Ivić d'avoir attiré mon attention sur les travaux de E. J. Scourfield. Je remercie E. J. Scourfield et M. Balazard d'avoir lu ce travail et soulevé certaines difficultés.

2. Notations. On désigne par $p$ (respectivement par $n$ ) un nombre premier (respectivement un entier) générique. On pose, pour $g \in G, f(n)=$ $n g(n)$ et

$$
N(x)=\sum_{f(n) \leq x} 1, \quad M_{q}(x)=\sum_{\substack{f(n) \leq x \\(n, q)=1}} \mu^{2}(n)
$$

où $\mu$ est la fonction de Möbius. Si $n$ est sans facteur carré, on a

$$
g(n)=\prod_{p \mid n} g(p) .
$$

Nous poserons, pour $y$ tel que $2 \leq y \leq x$,

$$
\begin{gathered}
g(n, y)=\prod_{\substack{p \mid n \\
p \leq y}} g(p), \quad f(n, y)=n g(n, y), \\
M_{q}(x, y)=\sum_{\substack{f(n, y) \leq x \\
(n, q)=1}} \mu^{2}(n), \quad S_{q}(x, y)=\sum_{\substack{1 \leq n \leq x \\
(n, q)=1 \\
P_{-}(n)>y}} \mu^{2}(n) .
\end{gathered}
$$

On notera $P_{+}(n)$ (resp. $\left.P_{-}(n)\right)$ le plus grand (resp. le plus petit) facteur premier de $n$.

3. Démonstration du théorème 1 . Soit $n$ un entier $\geq 1$; écrivons $n=s q$ avec $(s, q)=1, s$ squarefree et $q$ squarefull. On a

$$
N(x)=\sum_{\substack{n=s q \\ f(s) f(q) \leq x \\(s, q)=1}} 1=\sum_{f(q) \leq x}\left(\sum_{\substack{f(s) \leq x / f(q) \\(s, q)=1}} 1\right)=\sum_{f(q) \leq x} M_{q}\left(\frac{x}{f(q)}\right) .
$$


Posons $d=1-b, 0<b<1 / 2$; donc $1 / 2<d<1$. On a

$$
N(x)=\sum_{f(q) \leq x^{d / 2}} M_{q}\left(\frac{x}{f(q)}\right)+\sum_{x^{d / 2}<f(q) \leq x} M_{q}\left(\frac{x}{f(q)}\right)=: \Sigma_{1}+\Sigma_{2} .
$$

Evaluons $\Sigma_{1}$. D'après la propriété $(3)$ on a $g(n) \gg n^{-b}$, donc $f(n) \gg n^{d}$. Mais comme $x^{d / 2} \geq f(q)$, alors $q \ll \sqrt{x}$ et par suite $q f(q) \leq q x^{d / 2} \ll$ $x^{(d+1) / 2} \ll x$, c'est-à-dire $q \ll x / f(q)$. D'où l'on obtient pour $x$ suffisamment grand,

$$
\log q \leq\left(\frac{1}{4} \log \frac{x}{f(q)}\right)^{2}
$$

Le théorème 2 s'applique donc à $\Sigma_{1}$ et donne

$$
\begin{aligned}
\Sigma_{1} & =x \sum_{f(q) \leq x^{d / 2}} \frac{B(q, g)}{f(q)} \\
& +O_{\varepsilon}\left(x \sum_{f(q) \leq x^{d / 2}} \frac{1}{f(q)} \exp \left(-(1-\varepsilon) \sqrt{\frac{\beta}{2} \log \left(\frac{x}{f(q)}\right) \log \log \left(\frac{x}{f(q)}\right)}\right)\right) .
\end{aligned}
$$

Posons

$$
c(q)=\prod_{p \mid q}\left(1+\frac{1}{p g(p)}\right)^{-1}, \quad \eta(n)= \begin{cases}1 & \text { si } n \text { est squarefull } \\ 0 & \text { sinon. }\end{cases}
$$

On a

$$
\sum_{q=1}^{\infty} \frac{c(q)}{f(q)}=\sum_{n=1}^{\infty} \frac{\eta(n) c(n)}{n g(n)}=\prod_{p}\left(1+\sum_{\alpha=2}^{\infty} \frac{c(p)}{p^{\alpha} g\left(p^{\alpha}\right)}\right) .
$$

Il s'ensuit que

$$
\begin{aligned}
\sum_{q=1}^{\infty} \frac{B(q, g)}{f(q)} & =\prod_{p}\left(1-\frac{1}{p}\right)\left(1+\frac{1}{p g(p)}\right)\left(1+\sum_{\alpha=2}^{\infty} \frac{c(p)}{p^{\alpha} g\left(p^{\alpha}\right)}\right) \\
& =\prod_{p}\left(1+\sum_{\alpha=1}^{\infty} p^{-\alpha}\left(\frac{1}{g\left(p^{\alpha}\right)}-\frac{1}{g\left(p^{\alpha-1}\right)}\right)\right):=A(g) .
\end{aligned}
$$

Montrons maintenant que

$\sum_{f(q)>x^{d / 2}} \frac{c(q)}{f(q)} \ll x^{\lambda}(\log x)^{k} \quad$ où $\lambda=(1-2 d) / 4,-1 / 4<\lambda<0 \quad$ et $\quad k \geq 1$.

Posons

$$
\sigma=\frac{1}{\log x}, \quad \theta=-1+\frac{1}{2 d}+\sigma, \quad \gamma=\frac{1+2 d \sigma}{2 d}
$$


On a, pour $x$ suffisamment grand,

$$
\sum_{f(q)>x^{d / 2}} \frac{c(q)}{f(q)} \leq x^{d \theta / 2} \sum_{q=1}^{\infty} \frac{1}{(f(q))^{1+\theta}} .
$$

Mais

$$
\begin{aligned}
\sum_{q=1}^{\infty} \frac{1}{(f(q))^{1+\theta}} & =\prod_{p}\left(1+\sum_{\alpha=2}^{\infty} \frac{1}{\left(p^{\alpha} g\left(p^{\alpha}\right)\right)^{\gamma}}\right) \leq \prod_{p}\left(1+\frac{k}{p^{1+2 d \sigma}}\right) \\
& \ll \zeta^{k}(1+2 d \sigma) \ll(\log x)^{k},
\end{aligned}
$$

$k$ étant supposée entier. D'où l'on obtient le résultat annoncé.

Posons

$$
u=\log x \quad \text { et } \quad u_{q}=\log \left(\frac{x}{f(q)}\right),
$$

et remarquons que :

$$
\text { si } 0<f(q)<1 \quad \text { alors } u_{q} \geq u \text { et donc } u_{q} \log u_{q} \geq u \log u,
$$

et

$$
\text { si } \quad 1 \leq f(q) \leq x^{d / 2} \quad \text { alors } \quad u_{q} \leq u .
$$

On peut écrire dans ce dernier cas,

$$
\begin{aligned}
(u \log u)^{1 / 2}-\left(u_{q} \log u_{q}\right)^{1 / 2} & =\frac{u \log u-u_{q} \log u_{q}}{(u \log u)^{1 / 2}+\left(u_{q} \log u_{q}\right)^{1 / 2}} \\
& \leq \frac{\int_{u_{q}}^{u}(\log v+1) d v}{(u \log u)^{1 / 2}} \leq \frac{\log u+1}{(u \log u)^{1 / 2}} \log f(q) .
\end{aligned}
$$

Il s'ensuit que

$$
-\left(u_{q} \log u_{q}\right)^{1 / 2} \leq-(u \log u)^{1 / 2}+\frac{\log u+1}{(u \log u)^{1 / 2}} \log f(q) .
$$

Posant

$$
\sigma=\frac{\beta^{1 / 2}(\log u+1)}{(2 u \log u)^{1 / 2}}
$$

et appliquant ce qui précède, on obtient

$$
\begin{aligned}
\sum_{f(q) \leq x^{d / 2}} & \frac{1}{f(q)} \exp \left(-(1-\varepsilon)\left((\beta / 2) u_{q} \log u_{q}\right)^{1 / 2}\right) \\
\leq & \sum_{0<f(q)<1} \frac{1}{f(q)} \exp \left(-(1-\varepsilon)((\beta / 2) u \log u)^{1 / 2}\right) \\
& +\sum_{1 \leq f(q) \leq x^{d / 2}} \frac{1}{(f(q))^{1-\sigma}} \exp \left(-(1-\varepsilon)((\beta / 2) u \log u)^{1 / 2}\right)
\end{aligned}
$$




$$
\begin{aligned}
& \leq \exp \left(-(1-\varepsilon)((\beta / 2) u \log u)^{1 / 2}\right)\left(\sum_{q=1}^{\infty} \frac{1}{(f(q))^{1-\sigma}}+\sum_{q=1}^{\infty} \frac{1}{f(q)}\right) \\
& \ll \exp \left(-(1-\varepsilon)((\beta / 2) u \log u)^{1 / 2}\right),
\end{aligned}
$$

pour $x$ suffisamment grand. En effet, on a pour $\eta>1 /(2 d)$,

$$
\sum_{q=1}^{\infty} \frac{1}{(f(q))^{\eta}}=\prod_{p}\left(1+\sum_{\alpha=2}^{\infty} \frac{1}{\left(p^{\alpha} g\left(p^{\alpha}\right)\right)^{\eta}}\right) \leq \prod_{p}\left(1+\frac{1}{p^{2 d \eta}}\right) \ll 1
$$

puisque

$$
\sum_{\alpha=2}^{\infty} \frac{1}{p^{\alpha} g\left(p^{\alpha}\right)} \ll \frac{1}{p^{2 d}}
$$

Finalement,

$$
\begin{aligned}
\Sigma_{1}=A(g) x+O\left(x^{1+\lambda}(\log x)^{k}\right) & \\
& +O_{\varepsilon}(x \exp (-(1-\varepsilon) \sqrt{(\beta / 2) \log x \log \log x})) \\
=A(g) x+O_{\varepsilon}(x \exp (-(1-\varepsilon) \sqrt{(\beta / 2) \log x \log \log x}) & .
\end{aligned}
$$

Evaluons maintenant

$$
\Sigma_{2}=\sum_{x^{d / 2}<f(q) \leq x} M_{q}\left(\frac{x}{f(q)}\right) .
$$

On a

$$
\begin{aligned}
M_{q}\left(\frac{x}{f(q)}\right) & =\sum_{\substack{f(n) \leq x / f(q) \\
(n, q)=1}} \mu^{2}(n) \leq \sum_{f(n) \leq x / f(q)} \mu^{2}(n) \\
& =M_{1}\left(\frac{x}{f(q)}\right) \ll \frac{x}{f(q)}
\end{aligned}
$$

d'après le théorème 2. Par conséquent

$$
\begin{aligned}
\Sigma_{2} & \ll x \sum_{x^{d / 2}<f(q) \leq x} \frac{1}{f(q)} \ll x \sum_{f(q)>x^{d / 2}} \frac{1}{f(q)} \ll x^{1+\lambda} \log ^{k} x \\
& \ll x \exp (-(1-\varepsilon) \sqrt{(\beta / 2) \log x \log \log x}) .
\end{aligned}
$$

Ceci termine la preuve du théorème 1 .

\section{Lemmes nécessaires}

Lemme 1. Soit A une constante positive. On a, pour $x$ suffisamment grand et $y^{\delta}>16 A \log x$,

$$
M_{q}\left(x \exp \left(-\frac{16 A \log x}{y^{\delta}}\right), y\right) \leq M_{q}(x) \leq M_{q}\left(x\left(1-\frac{6 A \log x}{y^{\delta}}\right)^{-1}, y\right) .
$$


Démonstration. Soit $g \in G$. D'après la propriété (2), il existe une constante $A>0$ telle que pour tout nombre premier $p$ on ait

$$
1-A / p^{\delta} \leq g(p) \leq 1+A / p^{\delta} .
$$

Soit $m$ un entier $\geq 1$ sans facteur carré. On a

$$
g(m)=g(m, y) \prod_{\substack{p \mid m \\ p>y}} g(p) .
$$

Posons

$$
\Pi(m, y):=\prod_{\substack{p \mid m \\ p>y}} g(p)
$$

Minorons $\Pi(m, y)$ :

$$
\begin{aligned}
\Pi(m, y) & =\prod_{\substack{p \mid m \\
p>y}} g(p) \geq \prod_{\substack{p \mid m \\
p>y}}\left(1-\frac{A}{p^{\delta}}\right) \geq \prod_{p \mid m}\left(1-\frac{A}{y^{\delta}}\right) \geq\left(1-\frac{A}{y^{\delta}}\right)^{\omega(m)} \\
& \geq\left(1-\frac{A}{y^{\delta}}\right)^{2 \log m} \geq 1-\frac{2 A \log m}{y^{\delta}}
\end{aligned}
$$

pourvu que $y^{\delta}>A$. Maintenant d'après la propriété (3) définissant la classe $G$, on a

$$
f(m)=m g(m) \geq B m^{1-b}=B m^{d},
$$

avec $d=1-b, 1 / 2<d<1$. Il s'ensuit que si $f(m) \leq x$, alors $x \geq B m^{d}$, c'est-à-dire $m \leq(x / B)^{1 / d}$; alors

$$
\log m \leq 2 \log x-\log B \leq 3 \log x
$$

pour $x$ assez grand, d'où

$$
\Pi(m, y) \geq 1-\frac{6 A \log x}{y^{\delta}} .
$$

Finalement, on a

$$
f(m) \geq m g(m, y)\left(1-\frac{6 A \log x}{y^{\delta}}\right)
$$

pour $x$ assez grand et $y^{\delta}>6 A \log x$, d'où l'on obtient

$$
M_{q}(x) \leq M_{q}\left(x\left(1-\frac{6 A \log x}{y^{\delta}}\right)^{-1}, y\right) .
$$

Majoration de $\Pi(m, y)$ :

$$
\Pi(m, y)=\prod_{\substack{p \mid m \\ p>y}} g(p) \leq \prod_{\substack{p \mid m \\ p>y}}\left(1+\frac{A}{p^{\delta}}\right) \leq \prod_{p \mid m}\left(1+\frac{A}{y^{\delta}}\right) \leq\left(1+\frac{A}{y^{\delta}}\right)^{\omega(m)}
$$




$$
\begin{aligned}
& \leq\left(1+\frac{A}{y^{\delta}}\right)^{2 \log m}=\exp \left(2 \log m \log \left(1+\frac{A}{y^{\delta}}\right)\right) \\
& \leq \exp \left(\frac{2 A \log m}{y^{\delta}}\right) .
\end{aligned}
$$

Donc

$$
\Pi(m, y) \leq \exp \left(\frac{2 A \log m}{y^{\delta}}\right)
$$

Supposons maintenant que

$$
y^{\delta}>8 A \quad \text { et } \quad f(m, y):=m g(m, y) \leq x \exp \left(-\frac{16 A \log x}{y^{\delta}}\right)
$$

alors, on a

$$
\begin{aligned}
m^{d} & \ll f(m) \leq f(m, y) \exp \left(\frac{2 A \log m}{y^{\delta}}\right) \\
& \leq f(m, y) \exp \left(\log m^{1 / 4}\right)=f(m, y) m^{1 / 4} \\
& \leq x \exp \left(-\frac{16 A \log m}{y^{\delta}}\right) m^{1 / 4} \leq x m^{1 / 4},
\end{aligned}
$$

d'où $x \geq B m^{d-1 / 4}=B m^{\tau}, \tau=d-\frac{1}{4}=\frac{3}{4}-b \geq \frac{1}{4}$ et donc

$$
\log x \geq \tau \log m+\log B \geq \frac{1}{4} \log m+\log B
$$

$(0<B \leq 1$ car $1=g(1) \geq B \cdot 1=B)$ et donc pour $x$ assez grand, $\log m \leq 8 \log x$. Par conséquent,

$$
\begin{aligned}
f(m) & =f(m, y) \Pi(m, y) \leq f(m, y) \exp \left(\frac{2 A \log m}{y^{\delta}}\right) \\
& \leq f(m, y) \exp \left(\frac{16 A \log x}{y^{\delta}}\right) \\
& \leq x \exp \left(-\frac{16 A \log x}{y^{\delta}}\right) \cdot \exp \left(\frac{16 A \log x}{y^{\delta}}\right)=x .
\end{aligned}
$$

Donc, si $y^{\delta}>8 A$ et $x$ est suffisamment grand, on a

$$
M_{q}\left(x \exp \left(-\frac{16 A \log x}{y^{\delta}}\right), y\right) \leq M_{q}(x) .
$$

Ceci termine la preuve du lemme.

Lemme 2. Soient y et $k$ des nombres réels tels que $0 \leq k<\beta \log y$, et soit $g \in G$. Posons

$$
\sigma=1-\frac{k}{\log y} \quad \text { et } \quad f(n)=n g(n) .
$$


Alors, on a

$$
\sum_{\substack{n \geq 1 \\ P_{+}(n) \leq y}} \frac{\mu^{2}(n)}{(f(n))^{\sigma}} \ll \log y \exp \left(O\left(e^{k}\right)\right) .
$$

Démonstration. On a

$$
\begin{aligned}
\sum_{\substack{n \geq 1 \\
P_{+}(n) \leq y}} \frac{\mu^{2}(n)}{(f(n))^{\sigma}} & =\prod_{p \leq y}\left(1+\frac{1}{p^{\sigma}(g(p))^{\sigma}}\right)=\exp \left(\sum_{p \leq y} \log \left(1+\frac{1}{p^{\sigma}(g(p))^{\sigma}}\right)\right) \\
& \leq \exp \left(\sum_{p \leq y} \frac{1}{p^{\sigma}(g(p))^{\sigma}}\right) .
\end{aligned}
$$

Comme $g(p)=1+O\left(p^{-\delta}\right), \delta>0$, alors, pour $\sigma>0$,

$$
(g(p))^{\sigma}=\left(1+O\left(p^{-\delta}\right)\right)^{\sigma}=1+O\left(p^{-\delta}\right)
$$

et donc

$$
\frac{1}{p^{\sigma}(g(p))^{\sigma}}=p^{-\sigma}\left(1+O\left(p^{-\delta}\right)\right)=p^{-\sigma}+O\left(p^{-(\delta+\sigma)}\right) .
$$

On obtient

$$
\sum_{p \leq y} \frac{1}{p^{\sigma}(g(p))^{\sigma}}=\sum_{p \leq y} p^{-\sigma}+O\left(\sum_{p \leq y} p^{-(\delta+\sigma)}\right)=\sum_{p \leq y} p^{-\sigma}+O(1),
$$

pourvu que $\sigma>0$ et $\sigma>1-\beta$. Mais

$$
\sum_{p \leq y} p^{-\sigma} \leq \log \log y+O\left(e^{k}\right)
$$

il s'ensuit que

$$
\sum_{n=1}^{\infty} \frac{\mu^{2}(n)}{(f(n))^{\sigma}} \ll \log y \exp \left(O\left(e^{k}\right)\right)
$$

pourvu que $\sigma>0$ et $\sigma>1-\beta$.

Lemme 3. Soient $2 \leq y \leq x$ des nombres réels. Posons $u=\log x / \log y$. Supposons que $u<y^{\beta}$. Alors on a

$$
\sum_{\substack{f(n) \leq x \\ P_{+}(n) \leq y}} \mu^{2}(n) \ll x \log y \exp (-u \log u+O(u)) .
$$

Démonstration. On a, pour tout $\sigma>0$,

$$
\sum_{\substack{f(n) \leq x \\ P_{+}(n) \leq y}} \mu^{2}(n) \leq x^{\sigma} \sum_{\substack{n=1 \\ P_{+}(n) \leq y}}^{\infty} \frac{\mu^{2}(n)}{(f(n))^{\sigma}} .
$$

On pose $\sigma=1-\log u / \log y$; le résultat suit alors de l'application du lemme 2 . 
Lemme 4 ([3], [8]). Soient $x, y, u$ des nombres réels tels que $3 \leq y \leq x$, et $u=\log x / \log y$. Si $\log y>\sqrt{\log x}$, alors

$$
\Phi(x, y):=\sum_{\substack{n \leq x \\ P_{-}(n)>y}} 1=x \prod_{p \leq y}\left(1-\frac{1}{p}\right)\left\{1+O_{\varepsilon}(\exp (-(1-\varepsilon) u \log u))\right\}
$$

où $\varepsilon$ est un nombre réel positif arbitraire.

Lemme 5. Soient $x, y, u$ des nombres réels tels que $3 \leq y \leq x$ et $u=\log x / \log y$. Supposons que $u<\log y$. Alors pour $x$ et $x / y$ suffisamment grands, on a, uniformément pour tout $q \geq 1$,

$$
\begin{aligned}
S_{q}(x, y)=x \prod_{p \leq y}\left(1-\frac{1}{p}\right)\{ & \prod_{\substack{p \mid q \\
p>y}}\left(1-\frac{1}{p}\right) \prod_{\substack{p \nmid q \\
p>y}}\left(1-\frac{1}{p^{2}}\right) \\
& +O\left(\frac{\log x}{\sqrt{x / y}} \prod_{p \mid q}\left(1+\frac{1}{\sqrt{p}}\right)\right) \\
& \left.+O_{\varepsilon}\left(\exp \left(-(1-\varepsilon) u \log u+2 e u \frac{\log q}{y}\right)\right)\right\}
\end{aligned}
$$

où $\varepsilon$ est un nombre réel positif arbitraire.

Démonstration. Soit $\lambda(n, q)$ la fonction multiplicative définie par

$$
\lambda(1, q)=1, \quad \lambda\left(p^{\alpha}, q\right)= \begin{cases}-1 & \text { si } \alpha=1 \text { et } p \mid q \text { ou } \alpha=2 \text { et } p \nmid q, \\ 0 & \text { ailleurs. }\end{cases}
$$

Alors on a l'identité

$$
\sum_{d \mid n} \lambda(d, q)= \begin{cases}\mu^{2}(n) & \text { si }(n, q)=1 \\ 0 & \text { si }(n, q)>1\end{cases}
$$

On a

$$
\begin{aligned}
S_{q}(x, y) & =\sum_{\substack{1 \leq n \leq x \\
(n, q)=1 \\
P_{-}(n)>y}} \mu^{2}(n)=\sum_{\substack{n \leq x \\
P_{-}(n)>y}}\left(\sum_{d \mid n} \lambda(d, q)\right) \\
& =\sum_{\substack{d \leq x \\
P_{-}(d)>y}} \lambda(d, q)\left(\sum_{\substack{n \leq x \\
P_{-}(n)>y \\
d \mid n}} 1\right)=\sum_{\substack{d \leq x \\
P_{-}(d)>y}} \lambda(d, q)\left(\sum_{\substack{m \leq x / d \\
P_{-}(m)>y}} 1\right) \\
& =\sum_{\substack{d \leq x \\
P_{-}(d)>y}} \lambda(d, q) \Phi(x / d, y) .
\end{aligned}
$$


Appliquons maintenant le lemme 4. Posons

$$
u_{d}=\frac{\log (x / d)}{\log y} .
$$

On obtient, pour $u<\log y$,

$$
S_{q}(x, y)=x \prod_{p \leq y}\left(1-\frac{1}{p}\right) \sum_{\substack{d \leq x / y \\ P_{-}(d)>y}} \frac{\lambda(d, q)}{d}\left\{1+O_{\varepsilon}\left(\exp \left(-(1-\varepsilon) u_{d} \log u_{d}\right)\right)\right\} .
$$

Ecrivons

$$
\sum_{\substack{d \leq x / y \\ P_{-}(d)>y}} \frac{\lambda(d, q)}{d}=\sum_{\substack{d \geq 1 \\ P_{-}(d)>y}} \frac{\lambda(d, q)}{d}-\sum_{\substack{d>x / y \\ P_{-}(d)>y}} \frac{\lambda(d, q)}{d} .
$$

On a

$$
\begin{aligned}
\sum_{\substack{d \geq 1 \\
P_{-}(d)>y}} \frac{\lambda(d, q)}{d} & =\prod_{p>y}\left(1+\sum_{\alpha=1}^{\infty} \frac{\lambda\left(p^{\alpha}, q\right)}{p^{\alpha}}\right) \\
& =\prod_{\substack{p \mid q \\
p>y}}\left(1-\frac{1}{p}\right) \prod_{\substack{p \nmid q \\
p>y}}\left(1-\frac{1}{p^{2}}\right)
\end{aligned}
$$

et

$$
\left|-\sum_{\substack{d>x / y \\ P_{-}(d)>y}} \frac{\lambda(d, q)}{d}\right| \leq \sum_{\substack{d>x / y \\ P_{-}(d)>y}} \frac{|\lambda(d, q)|}{d} \leq \sum_{d>x / y} \frac{|\lambda(d, q)|}{d} .
$$

Posant $\sigma=1 / \log (x / y)$, on obtient, pour $x / y>e^{2}$,

$$
\begin{aligned}
\sum_{d>x / y} \frac{|\lambda(d, q)|}{d} & \leq(x / y)^{-1 / 2+\sigma} \sum_{d=1}^{\infty} \frac{|\lambda(d, q)|}{d^{1 / 2+\sigma}} \\
& \leq(x / y)^{-1 / 2+\sigma} \prod_{p \mid q}\left(1+\frac{1}{p^{1 / 2+\sigma}}\right) \prod_{p \nmid q}\left(1+\frac{1}{p^{1+2 \sigma}}\right) \\
& \leq e(x / y)^{-1 / 2} \prod_{p \mid q}\left(1+\frac{1}{p^{1 / 2+\sigma}}\right) \prod_{p}\left(1+\frac{1}{p^{1+2 \sigma}}\right) \\
& \leq e(x / y)^{-1 / 2} \prod_{p \mid q}\left(1+\frac{1}{p^{1 / 2+\sigma}}\right) \zeta(1+2 \sigma) \\
& \ll(x / y)^{-1 / 2} \log ^{\log (x / y)} \prod_{p \mid q}\left(1+\frac{1}{\sqrt{p}}\right)
\end{aligned}
$$




$$
\ll(x / y)^{-1 / 2} \log x \prod_{p \mid q}\left(1+\frac{1}{\sqrt{p}}\right),
$$

lorsque $x / y$ tend vers l'infini. Ecrivons

$$
\begin{aligned}
u \log u-u_{d} \log u_{d} & =\int_{u_{d}}^{u}(\log v+1) d v \\
& \leq(\log u+1)\left(u-u_{d}\right) \leq(\log u+1) \frac{\log d}{\log y} .
\end{aligned}
$$

Considérons

$$
\begin{aligned}
& \sum_{\substack{d \leq x / y \\
P_{-}(d)>y}} \frac{|\lambda(d, q)|}{d} \exp \left(-(1-\varepsilon) u_{d} \log u_{d}\right) \\
& \quad \leq \exp (-(1-\varepsilon) u \log u) \sum_{\substack{d \leq x \\
P_{-}(d)>y}} \frac{|\lambda(d, q)|}{d} \exp \left(\frac{\log d}{\log y}(\log u+1)\right) .
\end{aligned}
$$

On a

$$
\sum_{\substack{d \leq x \\ P_{-}(d)>y}} \frac{|\lambda(d, q)|}{d} \exp \left(\frac{\log u+1}{\log y} \log d\right)=\sum_{\substack{d \leq x \\ P_{-}(d)>y}} \frac{|\lambda(d, q)|}{d^{1-\sigma}} .
$$

On peut supposer

$$
\sigma=\frac{\log u+1}{\log y} \leq 1 / 4 .
$$

On a

$$
\sum_{\substack{d \leq x \\ P_{-}(d)>y}} \frac{|\lambda(d, q)|}{d^{1-\sigma}} \leq \sum_{\substack{d=1 \\ P_{-}(d)>y}}^{\infty} \frac{|\lambda(d, q)|}{d^{1-\sigma}}=\prod_{\substack{p \mid q \\ p>y}}\left(1+\frac{1}{p^{1-\sigma}}\right) \prod_{\substack{p \nmid q \\ p>y}}\left(1+\frac{1}{p^{2-2 \sigma}}\right) ;
$$

mais $2-2 \sigma \geq 3 / 2$, alors

$$
\sum_{\substack{d \leq x \\ P_{-}(d)>y}} \frac{|\lambda(d, q)|}{d^{1-\sigma}} \leq \prod_{\substack{p \mid q \\ p>y}}\left(1+\frac{1}{p^{1-\sigma}}\right) .
$$

Comme

$$
\begin{aligned}
\prod_{\substack{p \mid q \\
p>y}}\left(1+\frac{1}{p^{1-\sigma}}\right) & \leq \prod_{p \mid q}\left(1+\frac{1}{y^{1-\sigma}}\right) \leq\left(1+\frac{1}{y^{1-\sigma}}\right)^{\omega(q)} \\
& \leq\left(1+\frac{1}{y^{1-\sigma}}\right)^{2 \log q}=\exp \left(2 \log q \log \left(1+\frac{1}{y^{1-\sigma}}\right)\right)
\end{aligned}
$$




$$
\leq \exp \left(\frac{2 \log q}{y^{1-\sigma}}\right)
$$

et

$$
\begin{aligned}
y^{\sigma-1}=\exp ((\sigma-1) \log y) & =\exp \left(\left(\frac{\log u+1}{\log y}-1\right) \log y\right) \\
& =\exp (\log u+1-\log y)=e u / y,
\end{aligned}
$$

on obtient finalement

$$
\sum_{\substack{d \leq x / y \\ P_{-}(d)>y}} \frac{|\lambda(d, q)|}{d} \exp \left(-(1-\varepsilon) u_{d} \log u_{d}\right) \ll \exp \left(-(1-\varepsilon) u \log u+2 e u \frac{\log q}{y}\right) ;
$$

ceci termine la preuve du lemme.

Lemme 6. Soient $x, y, u$ des nombres réels tels que $x \geq y>\exp (\sqrt{\log x})$ et $u=\log x / \log y$. Pour $x$ et $x / y$ suffisamment grands, on a, uniformément pour $0 \leq \log q \leq y$,

$$
\begin{aligned}
& M_{q}(x, y)=x \prod_{p \nmid q}\left(1-\frac{1}{p^{2}}\right) \prod_{\substack{p \leq y \\
p \nmid q}}\left(1-\frac{1}{p^{2}}\right)^{-1} \\
& \quad \times \prod_{p \mid q}\left(1-\frac{1}{p}\right) \prod_{\substack{p \leq y \\
p \nmid q}}\left(1-\frac{1}{p}\right)\left(1+\frac{1}{p g(p)}\right) \\
& +O\left(\sqrt{x y} \log x \prod_{p \mid q}\left(1+\frac{1}{\sqrt{p}}\right)\right)+O_{\varepsilon}(x \log y \exp (-(1-\varepsilon) u \log u))
\end{aligned}
$$

où $\varepsilon$ est un nombre réel positif arbitraire.

Démonstration. Soit $n \geq 1$ un entier sans facteur carré. $n$ s'écrit

$$
n=k l \quad \text { avec } \quad(k, l)=1, P_{+}(k) \leq y \quad \text { et } \quad l=1 \quad \text { ou } \quad P_{-}(l)>y .
$$

Donc

$$
g(n, y)=g(k l, y)=g(k)
$$

et

$$
f(n, y)=f(k l, y)=k l g(k l, y)=f(k) l .
$$

Il s'ensuit alors que

$$
M_{q}(x, y)=\sum_{\substack{f(k) \leq x / y \\(k, q)=1 \\ P_{+}(k) \leq y}}^{\prime}\left(\sum_{\substack{1 \leq l \leq x / f(k) \\(l, q)=1 \\ P_{-}(l)>y}}^{\prime} 1\right)+O\left(\sum_{\substack{f(k) \leq x \\ P_{+}(k) \leq y \\(k, q)=1}}^{\prime} 1\right)
$$


où $\sum^{\prime}$ signifie que la somme porte sur les entiers squarefree. On a

$$
\sum_{\substack{f(k) \leq x \\ P_{+}(k) \leq y \\(k, q)=1}}^{\prime} 1 \leq \sum_{\substack{f(k) \leq x \\ P_{+}(k) \leq y}}^{\prime} 1 \ll_{\varepsilon} x \log y \exp (-(1-\varepsilon) u \log u)
$$

d'après le lemme 3. Examinons, maintenant, la quantité

$$
\sum_{\substack{f(k) \leq x / y \\ P_{+}(k) \leq y \\(k, q)=1}}^{\prime}\left(\sum_{\substack{1 \leq l \leq x / f(k) \\(l, q)=1 \\ P_{-}(l)>y}}^{\prime} 1\right)=\sum_{\substack{f(k) \leq x / y \\(k, q)=1 \\ P_{+}(k) \leq y}}^{\prime} S_{q}\left(\frac{x}{f(k)}, y\right) .
$$

Appliquons le lemme 5, et étudions chaque terme de la somme obtenue.

On a

$$
\begin{aligned}
& x \prod_{p \leq y}\left(1-\frac{1}{p}\right) \prod_{\substack{p \mid q \\
p>y}}\left(1-\frac{1}{p}\right) \prod_{\substack{p \nmid q \\
p>y}}\left(1-\frac{1}{p^{2}}\right) \sum_{\substack{f(k) \leq x / y \\
(k, q)=1 \\
P_{+}(k) \leq y}}^{\prime} \frac{1}{f(k)} \\
& =x \prod_{p \nmid q}\left(1-\frac{1}{p^{2}}\right) \prod_{\substack{p \nmid q \\
p \leq y}}\left(1-\frac{1}{p^{2}}\right)^{-1} \prod_{p \mid q}\left(1-\frac{1}{p}\right) \prod_{\substack{p \leq y \\
p \nmid q}}\left(1-\frac{1}{p}\right) \sum_{\substack{f(k) \leq x / y \\
(k, q)=1 \\
P_{+}(k) \leq y}}^{\prime} \frac{1}{f(k)} .
\end{aligned}
$$

Mais

$$
\sum_{\substack{f(k) \leq x / y \\(k, q)=1 \\ P_{+}(k) \leq y}}^{\prime} \frac{1}{f(k)}=\sum_{\substack{k=1 \\(k, q)=1 \\ P_{+}(k) \leq y}}^{\infty} \frac{1}{f(k)}-\sum_{\substack{f(k)>x / y \\(k, q)=1 \\ P_{+}(k) \leq y}}^{\prime} \frac{1}{f(k)}
$$

et

$$
\sum_{\substack{k=1 \\(k, q)=1 \\ P_{+}(k) \leq y}}^{\infty} \frac{1}{f(k)}=\prod_{\substack{p \leq y \\ p \nmid q}}\left(1+\frac{1}{p g(p)}\right) .
$$

Posons $\sigma=\log u / \log y$. On a

$$
\begin{aligned}
\sum_{\substack{f(k)>x / y \\
(k, q)=1 \\
P_{+}(k) \leq y}}^{\prime} \frac{1}{f(k)} & \leq \sum_{\substack{f(k)>x / y \\
P_{+}(k) \leq y}}^{\prime} \frac{1}{f(k)} \leq\left(\frac{y}{x}\right)^{\sigma} \sum_{\substack{k=1 \\
P_{+}(k) \leq y}}^{\infty}(f(k))^{\sigma-1} \\
& \ll_{\varepsilon} \log y \exp (-(1-\varepsilon) u \log u)
\end{aligned}
$$


par application du lemme 2. On a

$$
\begin{aligned}
x(x / y)^{-1 / 2} \log x \prod_{p \mid q} & \left(1+\frac{1}{\sqrt{p}}\right) \sum_{\substack{f(k) \leq x / y \\
(k, q)=1 \\
P_{+}(k) \leq y}}^{\prime} \frac{1}{f(k)} \\
& \leq(x y)^{1 / 2} \log x \prod_{p \mid q}\left(1+\frac{1}{\sqrt{p}}\right) \sum_{\substack{k=1 \\
P_{+}(k) \leq y \\
(k, q)=1}}^{\infty}(f(k))^{-1} \\
& \leq(x y)^{1 / 2} \log x \prod_{p \mid q}\left(1+\frac{1}{\sqrt{p}}\right) \prod_{\substack{p \leq y \\
p \nmid q}}\left(1+\frac{1}{p g(p)}\right) \\
& \leq(x y)^{1 / 2} \log x \prod_{p \mid q}\left(1+\frac{1}{\sqrt{p}}\right) \prod_{\substack{p \leq y \\
p \leq y}}\left(1+\frac{1}{p g(p)}\right) .
\end{aligned}
$$

Considérons maintenant

$$
\sum_{\substack{1 \leq f(k) \leq x / y \\(k, q)=1 \\ P_{+}(k) \leq y}}^{\prime} \frac{1}{f(k)} \exp \left(-(1-\varepsilon) u_{k} \log u_{k}+2 e u_{k}(\log q) / y\right):=\Sigma_{1}
$$

où

$$
u_{k}=\frac{\log (x / f(k))}{\log y} \leq u
$$

Mais

$$
-u_{k} \log u_{k} \leq-u \log u+\frac{\log u+1}{\log y} \log f(k) .
$$

Posant $\sigma=(\log u+1) / \log y$, on obtient

$$
\begin{aligned}
\Sigma_{1} & \leq \exp (-(1-\varepsilon) u \log u+2 e u(\log q) / y) \sum_{\substack{k=1 \\
P_{+}(k) \leq y}}^{\infty}(f(k))^{\sigma-1} \\
& \ll_{\varepsilon} \log y \exp (-(1-2 \varepsilon) u \log u+2 e u(\log q) / y) \\
& \ll_{\varepsilon} \log y \exp (-(1-2 \varepsilon) u \log u+2 e u)
\end{aligned}
$$

par application du lemme 2 et le fait que $(\log q) / y \leq 1$. Maintenant

$$
\Sigma_{2}:=\sum_{\substack{0<f(k)<1 \\(k, q)=1 \\ P_{+}(k) \leq y}}^{\prime} \frac{1}{f(k)} \exp \left(-(1-\varepsilon) u_{k} \log u_{k}+2 e u_{k}(\log q) / y\right)
$$




$$
\leq \sum_{\substack{0<f(k)<1 \\(k, q)=1 \\ P_{+}(k) \leq y}}^{\prime} \frac{1}{f(k)} \exp \left(-(1-\varepsilon) u \log u+2 e u_{k}\right)
$$

car $0 \leq(\log q) / y \leq 1$, et $u_{k} \geq u$. Donc

$$
\Sigma_{2} \leq \exp (-(1-\varepsilon) u \log u+2 e u) \sum_{\substack{0<f(k)<1 \\(k, q)=1 \\ P_{+}(k) \leq y}}^{\prime} \frac{1}{(f(k))^{1+\sigma}}
$$

où $\sigma=2 e / \log y$. Mais

$$
\sum_{\substack{0<f(k)<1 \\(k, q)=1 \\ P_{+}(k) \leq y}}^{\prime} \frac{1}{(f(k))^{1+\sigma}} \leq \prod_{p}\left(1+\frac{1}{(p g(p))^{1+\sigma}}\right) \leq \prod_{p}\left(1+\frac{k}{p^{1+\sigma}}\right)
$$

$$
\ll \zeta^{k}(1+\sigma) \ll(\log y)^{k} .
$$

Il vient alors que

$$
\Sigma_{2} \ll(\log y)^{k} \exp (-(1-2 \varepsilon) u \log u),
$$

et finalement

$$
\sum_{\substack{f(k) \leq x / y \\(k, q)=1}}^{\prime} \frac{1}{f(k)} \exp \left(-(1-\varepsilon) u_{k} \log u_{k}+2 e u_{k}(\log q) / y\right)
$$

$$
\ll \log ^{k} y \exp (-(1-2 \varepsilon) u \log u)
$$

uniformément pour $0 \leq \log q \leq y$. Enfin, remarquant que

$$
\prod_{p \leq y}\left(1-\frac{1}{p}\right) \ll \frac{1}{\log y}
$$

on obtient le résultat annoncé en regroupant les quantités estimées.

5. Démonstration du théorème 2. Remarquons que

$$
\begin{gathered}
\prod_{p \nmid q}\left(1-\frac{1}{p^{2}}\right) \prod_{\substack{p \nmid q \\
p \leq y}}\left(1-\frac{1}{p^{2}}\right)^{-1} \prod_{p \mid q}\left(1-\frac{1}{p}\right) \prod_{\substack{p \nmid q \\
p \leq y}}\left(1-\frac{1}{p}\right)\left(1+\frac{1}{p g(p)}\right) \\
=\prod_{p}\left(1-\frac{1}{p}\right)\left(1+\frac{1}{p g(p)}\right) \prod_{p \mid q}\left(1+\frac{1}{p g(p)}\right)^{-1} \\
\times \prod_{\substack{p \nmid q \\
p>y}}\left(1-\frac{1}{p^{2}}\right) \prod_{\substack{p \nmid q \\
p>y}}\left(1-\frac{1}{p}\right)^{-1}\left(1+\frac{1}{p g(p)}\right)^{-1}
\end{gathered}
$$


et

$$
\begin{gathered}
\prod_{\substack{p \nmid q \\
p>y}}\left(1-\frac{1}{p^{2}}\right)=1+O\left(\frac{1}{y}\right), \\
\prod_{\substack{p \nmid q \\
p>y}}\left(1-\frac{1}{p}\right)^{-1}\left(1+\frac{1}{p g(p)}\right)^{-1}=1+O\left(\frac{1}{y^{\beta}}\right)
\end{gathered}
$$

où $\beta=\min \{1, \delta\}$ et $\delta>0$. L'application des lemmes 1 et 6 donne, pour $x$ et $x / y$ suffisamment grands,

$$
\begin{aligned}
M_{q}(x)= & B(q, g) x+O\left(\sqrt{x y} \log x \prod_{p \mid q}\left(1+\frac{1}{\sqrt{p}}\right)\right) \\
& +O\left(\frac{x \log x}{y^{\beta}}\right)+O_{\varepsilon}\left(x \log ^{k} y \exp (-(1-\varepsilon) u \log u)\right)
\end{aligned}
$$

uniformément pour $q$ tel que $0 \leq \log q \leq y$. On choisit

$$
y=\exp (\sqrt{(1 /(2 \beta)) \log x \log \log x}) .
$$

Les hypothèses des lemmes 1 et 6 sont vérifiées et l'on a

$$
\begin{gathered}
u \log u=(1+o(1)) \sqrt{(\beta / 2) \log x \log \log x}, \\
\frac{\log x}{y^{\beta}} \leq \exp (-(1-\varepsilon) \sqrt{(\beta / 2) \log x \log \log x}) .
\end{gathered}
$$

Maintenant, sous la condition

$$
0 \leq \log q \leq\left(\frac{1}{4} \log x\right)^{2}
$$

on a

$$
\sqrt{x y} \log x \prod_{p \mid q}\left(1+\frac{1}{\sqrt{p}}\right)=O\left(\frac{x}{y^{(1-\varepsilon) \beta}}\right) .
$$

En effet, soit $p_{r}$ le rième nombre premier. Soit $r$ tel que

$$
\prod_{p \leq p_{r-1}} p<q \leq \prod_{p \leq p_{r}} p
$$

Alors le nombre de facteurs premiers de $q, \omega(q) \leq r$ et d'après le théorème des nombres premiers (dont on connait une démonstration élémentaire), on a

Maintenant,

$$
p_{r}=\log q\left(1+O\left(\frac{1}{\log \log q}\right)\right)
$$

$$
\prod_{p \mid q}\left(1+\frac{1}{\sqrt{p}}\right) \leq \prod_{p \leq p_{\omega(q)}}\left(1+\frac{1}{\sqrt{p}}\right) \leq \prod_{p \leq p_{r}}\left(1+\frac{1}{\sqrt{p}}\right) \leq \exp \left(\sum_{p \leq p_{r}} \frac{1}{\sqrt{p}}\right)
$$


et

$$
\sum_{p \leq p_{r}} \frac{1}{\sqrt{p}}=\frac{2 \sqrt{p_{r}}}{\log p_{r}}\left(1+O\left(\frac{1}{\log p_{r}}\right)\right)=\frac{2 \sqrt{\log q}}{\log \log q}\left(1+O\left(\frac{1}{\log \log q}\right)\right) .
$$

D'où

$$
\sqrt{x y} \log x \prod_{p \mid q}\left(1+\frac{1}{\sqrt{p}}\right) \ll \frac{x}{y^{(1-\varepsilon) \beta}} .
$$

Il s'ensuit que uniformément pour $q$ tel que $0 \leq \log q \leq\left(\frac{1}{4} \log x\right)^{2}$, on a

$$
M_{q}(x)=B(q, g) x+O_{\varepsilon}(x \exp (-(1-\varepsilon) \sqrt{(\beta / 2) \log x \log \log x})),
$$

pour tout $\varepsilon$ réel positif, ce qui termine la preuve du théorème 2 .

\section{Références}

[1] M. Balazard and A. Smati, Elementary proof of a theorem of Bateman, in: Analytic Number Theory, Proc. Conf. in Honor of Paul T. Bateman, Allerton Park, Ill., 1989, Progr. Math. 85, Birkhäuser, Boston 1990, 41-46.

[2] P. T. Bateman, The distribution of values of the Euler function, Acta Arith. 21 (1972), 329-345.

[3] H. Halberstam and H. E. Richert, Sieve Methods, Academic Press, 1974.

[4] A. Ivić, The distribution of values of some multiplicative functions, Publ. Inst. Math. (Beograd) (N.S.) 22(36) (1977), 87-94.

[5] E. J. Scourfield, On some sums involving the largest prime divisor of n, Acta Arith. 59 (1991), 339-363.

[6] A. Smati, Une formule asymptotique pour une classe de fonctions multiplicatives, Publ. Inst. Math. (Beograd) (N.S.) 49(63) (1990).

[7] -, Répartition des valeurs de la fonction d'Euler et de certaines fonctions multiplicatives, Thèse, Université de Limoges, 1990.

[8] G. Tenenbaum, Introduction à la théorie analytique et probabiliste des nombres, Publ. Inst. Elie Cartan 13, Université de Nancy, 1990.

DÉPARTEMENT DE MATHÉMATIQUES

FACULTÉ DES SCIENCES DE LIMOGES

123 AVENUE ALBERT THOMAS

87060 LIMOGES CEDEX, FRANCE 